\title{
Imaging Targets of the Sympathetic Nervous System of the Heart: Translational Considerations
}

\author{
Frank M. Bengel \\ Klinik für Nuklearmedizin, Medizinische Hochschule Hannover, Hannover, Germany
}

\begin{abstract}
The usefulness of imaging the cardiac sympathetic nervous system is increasingly supported by prospective clinical studies. The future success of this molecular imaging technique, however, will depend on careful design of additional trials. But preclinical and clinical use requires a thorough understanding of the underlying biology, which defines the relationship between neuronal tracer kinetics, disease mechanisms, and appropriate study interpretation. This review focuses on basic methodologic aspects considered relevant for successful continuation of the translation of cardiac neuronal imaging from a research tool toward a clinical test.
\end{abstract}

Key Words: molecular imaging; cardiovascular disease; autonomic nervous system; myocardial innervation

J Nucl Med 2011; 52:1167-1170

DOI: 10.2967/jnumed.110.084228

The first report on the use of radiolabeled catecholamine analogs to image cardiac sympathetic nerve terminals dates back 30 years (1). The initial introduction was followed by single-center preclinical and clinical studies, which suggested promise for characterizing arrhythmia and myocardial dysfunction. Multicenter clinical trials, however, have been conducted only recently $(2,3)$. They support a prognostic value in heart failure, and they mark an increasing interest on the part of the imaging community and represent an important step toward clinical application. The hope is that neuronal imaging will take a role in identifying heart failure patients who will benefit most from implantable devices or other costly therapies. Translational efforts in neuronal imaging are setting a precedent for the entire field of cardiovascular molecular imaging. The catecholamine analog ${ }^{123}$ I-metaiodobenzylguanidine ( $\left.{ }^{123} \mathrm{I}-\mathrm{MIBG}\right)$ is likely to become the next Food and Drug Administration-approved radiotracer for nonperfusion cardiac imaging, more than a decade after the approval of ${ }^{18} \mathrm{~F}-\mathrm{FDG}$ for viability (4). It is hoped that other molecular agents will follow. However, a critical issue in translation is maintaining balance

For correspondence or reprints contact: Frank M. Bengel, Klinik für Nuklearmedizin, Medizinische Hochschule Hannover, Carl-Neuberg-Strasse 1 D-30625 Hannover, Germany.

E-mail: bengel.frank@mh-hannover.de

COPYRIGHT @ 2011 by the Society of Nuclear Medicine, Inc. between the need for a robust, easy-to-obtain, yet accurate and unique marker on the one hand versus the danger of oversimplifying a complex biologic matter on the other hand. The former is necessary for clinical acceptance. The latter can be avoided only if previous knowledge from basic science is appropriately incorporated into translational study design.

\section{IMAGING TARGETS OF CARDIAC INNERVATION}

Figure 1 highlights the manifold pre- and postsynaptic mechanisms of sympathetic innervation that involve norepinephrine as a neurotransmitter. The kinetics of a radiolabeled norepinephrine analog are determined by those mechanisms in different ways, depending on its molecular properties $(5,6)$. Table 1 concisely summarizes radiolabeled catecholamines that have been applied to humans, along with key characteristics that determine their kinetic profiles.

\section{CONVENTIONAL SCINTIGRAPHIC ASSESSMENT}

The SPECT tracer ${ }^{123}$ I-MIBG is the most frequently used agent for imaging the cardiac sympathetic nervous system. Its prognostic value has been shown mostly by calculating a heart-to-mediastinum ratio from planar scans: a semiquantitative estimate of global myocardial catecholamine uptake $(2,3)$. Another parameter is the washout rate, a ratio of cardiac uptake between early and delayed images (7). Washout is often considered to reflect sympathetic tone. Although the clinical value of ${ }^{123} \mathrm{I}-\mathrm{MIBG}$ is increasingly well defined, there are some critical issues.

First, the heart-to-mediastinum ratio and washout rates are global markers of myocardial nerve integrity. They do not provide insights into regional heterogeneity. Heterogeneity, that is, impaired innervation in the viable border zone of an infarct, may be important for development of ventricular arrhythmia (8-10). Defect scores from ${ }^{123}$ I-MIBG SPECT studies may be used to interrogate this issue. But the scores should be interpreted with caution because the best-innervated region, which is set to $100 \%$ for normalization, may not be normal because of global downregulation of uptake. As occurs with balanced ischemia in perfusion SPECT (albeit much more likely in heart failure), a global reduction will result in underestimation of relative regional defect size. It is interesting that some studies suggest a regional defect score to be superior to the global heart-to-mediastinum ratio 


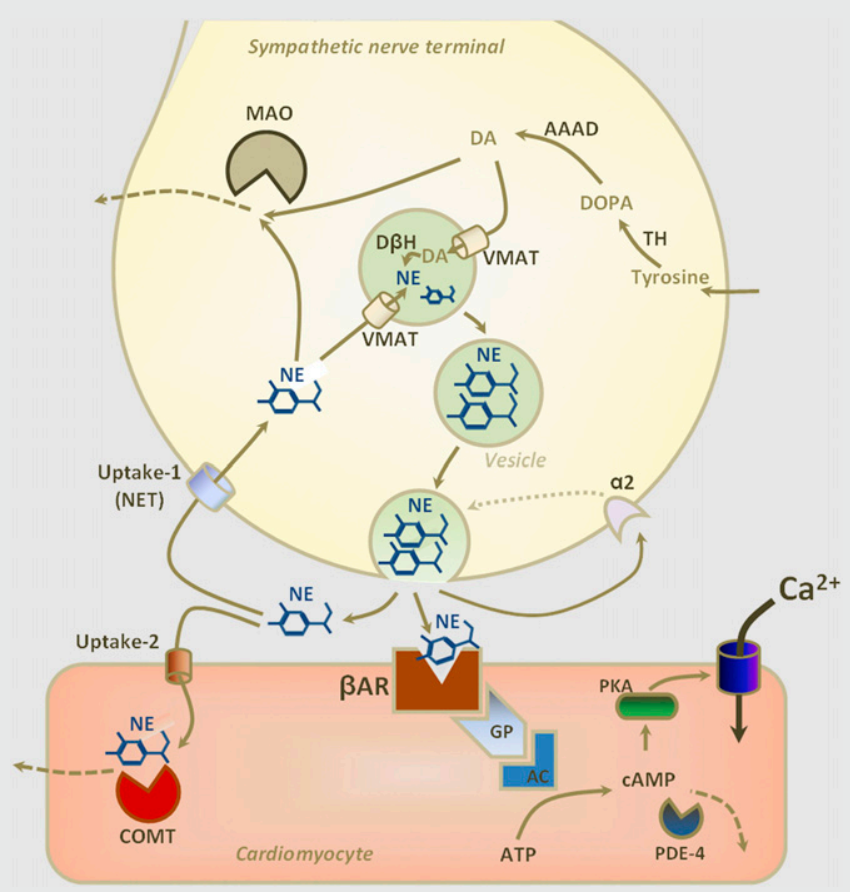

FIGURE 1. Molecular mechanisms of sympathetic neurotransmission. Norepinephrine (NE) is major neurotransmitter, which is released into synaptic cleft by exocytosis of storage vesicles on nerve firing. NE stimulates presynaptic $\alpha-2$ adrenoceptors, which inhibit vesicular exocytosis, and stimulates postsynaptic $\beta$-adrenoceptors ( $\beta A R)$. On postsynaptic side, activated $\beta A R$ increases levels of cyclic adenosine-monophosphate (cAMP) via G-proteins (GP) and adenylcyclase (AC). cAMP levels modulate electromechanical properties via activation of protein kinase A (PKA), which stimulates calcium influx into cardiomyocytes. Phosphodiesterase-4 (PDE-4) acts as major inhibitor of cAMP. On presynaptic side, clearance of NE from synaptic cleft occurs rapidly by reuptake into nerve terminals through norepinephrine transporter (NET; uptake-1). Once inside nerve terminals, $\mathrm{NE}$ is transported back into vesicles through vesicular monoamine transporter (VMAT) or is metabolized by MAO. Intraneuronal synthesis pathway for NE starts with tyrosine, which is converted to dihydroxyphenylalanine (DOPA) by tyrosine hydroxylase (TH). Aromatic L-amino acid decarboxylase (AAAD) then converts DOPA to dopamine (DA). DA is either metabolized by MAO or transported into vesicles by VMAT, where it is transformed to NE by dopamine- $\beta$-hydroxylase $(\mathrm{D} \beta \mathrm{H})$. Alternative pathway for NE clearance from synaptic cleft is uptake-2, which transports NE into tissue, where it is metabolized by catecholamine-O-methyl-transferase (COMT). ATP = adenosine triphosphate.

for prediction of arrhythmic events (11), thus implying that regional heterogeneity may be more important than global downregulation for development of arrhythmia.

Second, mechanisms underlying increased ${ }^{123}$ I-MIBG washout are particularly difficult to identify. Turnover within nerve terminals and spillover into the circulation, that is, increased sympathetic drive, may be contributors. But other factors, such as clearance from blood pool, or clearance through nonspecific uptake-2, especially in the setting of impaired uptake-1, may also play a role. Figure 2 highlights states that may influence catecholamine kinetics.

\section{CARDIAC NEURONAL PET}

PET, which enables absolute measurements of catecholamine retention, can be used for more detailed insights into cardiac neuronal biology. First, it is preferable for regional analysis. Every myocardial region, including infarct, border zone, and remote myocardium, can be quantitatively assessed. This ability helps to clarify the importance of regional heterogeneity versus globally impaired innervation. Of note, new ${ }^{18} \mathrm{~F}$-labeled tracers, such as ${ }^{18} \mathrm{~F}-\mathrm{LMI} 1195$, are under investigation and may become a commercial product in the future (12).

Another advantage of PET is that multiple tracers may be used. Single-tracer neuronal imaging provides only a snapshot of neuronal function. Combinations of tracers may provide deeper insights into nerve biology. As highlighted in Figure 2, reduced uptake of a catecholamine may be due to denervation or to impaired function of terminals. These conditions may differ in their likelihood to recover and may have different implications for the choice of therapy and for longterm outcome. If uptake of one catecholamine analog is reduced but another is avidly retained, impairment of a molecular mechanism that defines the kinetics of the first agent but not the second is likely, and true denervation is unlikely. ${ }^{11} \mathrm{C}$-epinephrine, for example, is sensitive to monoaminooxidase (MAO) degradation. If ${ }^{11} \mathrm{C}$-epinephrine is not stored efficiently in neuronal vesicles (where it is protected from MAO), retention may be diminished. Combination with another, MAO-resistant, agent such as ${ }^{11} \mathrm{C}$-hydroxyephedrine may then distinguish between true denervation (impaired retention of both agents; Fig. 2C) and functional impairment of vesicular storage (impaired retention of epinephrine and intact retention of the other tracer; Figs. 2D and 2E). Preliminary preclinical results suggest that the latter condition may exist, for example, in the viable infarct border zone (13).

Ultimately, the use of one or more PET agents may also help to improve the understanding of ${ }^{123}$ I-MIBG washout by identifying the contribution of specific neuronal uptake to the imaging signal at early and late imaging time points. This task, however, is challenging because of the shorter half-life of PET agents, which may show little washout during the early time window of roughly $60 \mathrm{~min}$ that can be used for imaging (14).

\section{INTERSPECIES INNERVATION DIFFERENCES}

Other important tracer properties include specific activity of the agent at the time of injection (lower specific activity will reduce myocardial tracer binding because of increased competition from cold compound), affinity for nonneuronal pathways, and interspecies differences.

In contrast to neuronal uptake-1, uptake-2 is responsible for catecholamine transport into nonneuronal tissue. The relative affinity of an agent, and the tissue expression of uptake- 1 versus uptake-2, will determine the neuronal specificity of the imaging signal-important when comparing results across different species. True changes of neuronal integrity may be masked by a strong contribution of nonspecific uptake-2 to the imaging 
TABLE 1

Clinically Used Radiolabeled Catecholamine Analogs

\begin{tabular}{|c|c|c|c|c|}
\hline Tracer & $\begin{array}{l}\text { Uptake-1 } \\
\text { affinity }\end{array}$ & $\begin{array}{l}\text { Vesicular } \\
\text { storage }\end{array}$ & $\begin{array}{l}\text { MAO/COMT- } \\
\text { sensitive }\end{array}$ & General characteristics \\
\hline 123|-MIBG & ++ & + & - & $\begin{array}{l}\text { Catecholamine analog; distributes in cytoplasm } \\
\text { and vesicles after neuronal uptake }\end{array}$ \\
\hline${ }^{11} \mathrm{C}-\mathrm{HED}$ & ++ & $\mathrm{O}$ & - & $\begin{array}{l}\text { Catecholamine analog; undergoes constant } \\
\text { uptake and reuptake by NET }\end{array}$ \\
\hline${ }^{11} \mathrm{C}$-EPI & + & ++ & + & $\begin{array}{l}\text { Physiologic neurotransmitter; requires vesicular } \\
\text { storage for protection from degradation }\end{array}$ \\
\hline${ }^{11} \mathrm{C}-\mathrm{PHEN}$ & + & + & + & $\begin{array}{l}\text { Catecholamine analog; washout reflects vesicular } \\
\text { leakage and MAO activity }\end{array}$ \\
\hline${ }^{18} \mathrm{~F}-\mathrm{FDA}$ & + & ++ & + & $\begin{array}{l}\text { Precursor of physiologic neurotransmitter; requires } \\
\text { enzymatic conversion and vesicular storage for } \\
\text { protection from degradation }\end{array}$ \\
\hline 18F-LMI1195 & $?$ & $?$ & $?$ & $\begin{array}{l}\text { Guanidine analog in phase } 1 \text { and } 2 \text { clinical studies; } \\
\text { published information is limited }\end{array}$ \\
\hline
\end{tabular}

COMT = catecholamine-O-methyl-transferase; HED = hydroxyephedrine; NET = norepinephrine transporter; EPI = epinephrine; PHEN = phenylephrine; FDA = fluorodopamine.

signal. Although rats and dogs seem to have significant uptake-2, it is almost absent in rabbits (15). Pigs seem to come closest to humans, with present but low levels of uptake-2.

Another issue across species is differences in catecholamine turnover and sympathetic tone. Some insights into this issue have been obtained using multiple PET agents in a rodent model. In healthy rats, ${ }^{11} \mathrm{C}$-hydroxyephedrine showed high myocardial uptake and sustained retention, whereas MAO-sensitive ${ }^{11} \mathrm{C}$-epinephrine showed moderate uptake and significant washout, which was similar to that of another MAO-sensitive agent, ${ }^{11} \mathrm{C}$-phenylephrine. A chase with desipramine after tracer injection resulted in increased washout only for ${ }^{11} \mathrm{C}$-hydroxyephedrine. These data support continuous uptake, release, and reuptake of MAO-

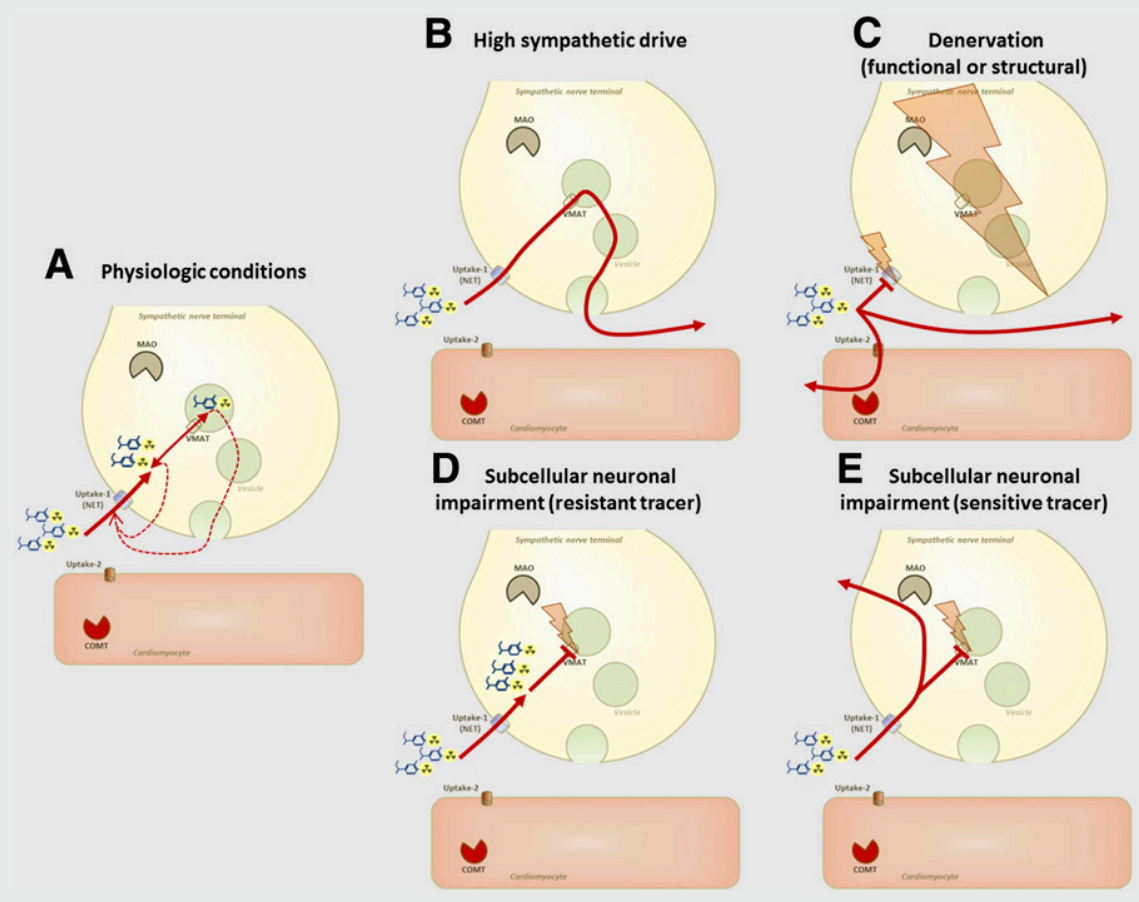

FIGURE 2. Simplified schematic display of various conditions of sympathetic nerve terminal and their effect on kinetics of radiolabeled catecholamines. (A) Physiologic condition with tracer storage due to intact neuronal uptake-1 and vesicular monoamine transporter (VMAT). Release due to nerve firing or transmembraneous loss is compensated by rapid reuptake. (B) High adrenergic drive. Rapid uptake is followed by rapid release due to nerve firing. Reuptake is not sufficient, resulting in washout into systemic circulation. (C) Functional (absence or dysfunctioning of uptake-1) or structural denervation (absence or loss of nerve terminal). Lack of specific reuptake into nerve terminals through norepinephrine transporter (NET; uptake-1) may cause nonspecific uptake2 by myocytes, eventually followed by metabolism through catecholamine-Omethyl-transferase (COMT). There is also washout into systemic circulation. (D and E) Situation in cases of subcellular nerve terminal dysfunction, that is, impaired vesicular storage. Tracer that is resistant

to metabolic degradation by neuronal MAO may show neuronal, extravesicular uptake (D), whereas tracer sensitive to MAO may be degraded and washed out (E). Situations B, C, and E all may contribute to washout of sympathetic neuronal tracer. Situations $D$ and E may be distinguished by PET using, for example, ${ }^{11} \mathrm{C}$-hydroxyephedrine (D) and ${ }^{11} \mathrm{C}$-epinephrine (E). 
TABLE 2

Other Molecular Radiotracers of Cardiac Autonomic Nervous System

\begin{tabular}{cll}
\hline Tracer & Preclinical/clinical & \multicolumn{1}{c}{ Target } \\
\hline${ }^{11} \mathrm{C}$-CGP12177 & Clinical & $\beta$-adrenoceptors \\
${ }^{11} \mathrm{C}$-CGP12388 & Clinical & $\beta$-adrenoceptors \\
${ }^{11} \mathrm{C}$-GB67 & Preclinical & $\alpha$-1 adrenoceptors \\
${ }^{11} \mathrm{C}$-rolipram & Preclinical & $\begin{array}{c}\text { Phosphodiesterase-4 } \\
\text { (downstream signaling) }\end{array}$ \\
& & Muscarinic receptors \\
${ }^{11} \mathrm{C}-$ MQNB & Clinical &
\end{tabular}

MQNB = methylquinuclidinyl benzilate.

resistant catecholamine analogs. But increased turnover of the physiologic neurotransmitter ${ }^{11} \mathrm{C}$-epinephrine suggests elevated sympathetic activity in the rodent model (16).

Hence, animal work may be used to generate hypotheses, but confirmation in human studies is recommended before larger trials are designed.

\section{BEYOND NERVE TERMINAL FUNCTION}

Combination of 2 or more autonomic nervous system tracers is attractive for obtaining detailed insights into the pathobiology or biology of cardiac innervation. A neuronal stress test has, for example, been proposed, using amitriptyline as a challenge before repeated ${ }^{123}$ I-MIBG imaging (17). This method may unmask reduced neuronal uptake reserve in subjects with movement disorders with an initially normal scan result. Additionally, tracers for several other molecular structures of the autonomic nervous system are under preclinical or early clinical investigation. Table 2 provides an overview. Combinations of autonomic imaging agents have, for example, been used to identify an imbalance between presynaptic neuronal integrity and postsynaptic receptor density in progressive heart failure (18), to show that postsynaptic receptor density and downstream signaling may be impaired despite intact presynaptic catecholamine uptake in adriamycin-induced cardiotoxicity (19) or to show that parasympathetic muscarinic receptors may be upregulated after myocardial infarction (20).

\section{CONCLUSION}

Although imaging of the cardiac autonomic nervous system has entered large-scale clinical trials that will ultimately lead to approval as a clinical tool, the basic science behind this methodology remains important. Specifics of the used tracers and preclinical animal models need to be considered for translation. Establishment of quantitative measures for global and regional innervation will be important. And novel tracers will be explored in order to resolve existing issues and provide further insights.

\section{ACKNOWLEDGMENTS}

Dr. Bengel is a recipient of research grants from GE Healthcare, Lantheus Medical Imaging, and Bracco Diagnostics. No other potential conflict of interest relevant to this article was reported.

\section{REFERENCES}

1. Raffel DM, Wieland DM. Development of mIBG as a cardiac innervation imaging agent. JACC Cardiovasc Imaging. 2010;3:111-116.

2. Agostini D, Verberne HJ, Burchert W, et al. I-123-mIBG myocardial imaging for assessment of risk for a major cardiac event in heart failure patients: insights from a retrospective European multicenter study. Eur J Nucl Med Mol Imaging. 2008:35:535-546

3. Jacobson AF, Senior R, Cerqueira MD, et al. Myocardial iodine-123 metaiodobenzylguanidine imaging and cardiac events in heart failure: results of the prospective ADMIRE-HF (AdreView Myocardial Imaging for Risk Evaluation in Heart Failure) study. J Am Coll Cardiol. 2010;55:2212-2221.

4. Bengel FM, Higuchi T, Javadi MS, Lautamaki R. Cardiac positron emission tomography. J Am Coll Cardiol. 2009;54:1-15.

5. Lautamaki R, Tipre D, Bengel FM. Cardiac sympathetic neuronal imaging using PET. Eur J Nucl Med Mol Imaging. 2007;34(suppl 1):S74-S85.

6. Raffel DM, Jung YW, Gildersleeve DL, et al. Radiolabeled phenethylguanidines: novel imaging agents for cardiac sympathetic neurons and adrenergic tumors. $J$ Med Chem. 2007;50:2078-2088.

7. Bengel FM, Schwaiger M. Assessment of cardiac sympathetic neuronal function using PET imaging. J Nucl Cardiol. 2004;11:603-616.

8. Matsunari I, Schricke U, Bengel FM, et al. Extent of cardiac sympathetic neuronal damage is determined by the area of ischemia in patients with acute coronary syndromes. Circulation. 2000;101:2579-2585.

9. Sasano T, Abraham MR, Chang KC, et al. Abnormal sympathetic innervation of viable myocardium and the substrate of ventricular tachycardia after myocardial infarction. J Am Coll Cardiol. 2008;51:2266-2275.

10. Simoes MV, Barthel P, Matsunari I, et al. Presence of sympathetically denervated but viable myocardium and its electrophysiologic correlates after early revascularised, acute myocardial infarction. Eur Heart J. 2004;25:551-557.

11. Boogers MJ, Borleffs CJ, Henneman MM, et al. Cardiac sympathetic denervation assessed with 123-iodine metaiodobenzylguanidine imaging predicts ventricular arrhythmias in implantable cardioverter-defibrillator patients. J Am Coll Cardiol. 2010;55:2769-2777.

12. Liu Y, Srivastiva A, Mulnix T, et al. Quantification of normal pattern of regional myocardial uptake of ${ }^{18} \mathrm{~F}$ LMI1195, a novel tracer for imaging myocardial sympathetic function: first-in-human study [abstract]. J Nucl Med. 2010;51(suppl 2):250P.

13. Higuchi T, Lautamaki R, Sasano $\mathrm{T}$, et al. Viable infarct borderzone shows impaired sympathetic innervation rather than true denervation: insights from a porcine model of myocardial infarction [abstract]. J Nucl Med. 2010;51(suppl 2):27P.

14. Schwaiger M, Kalff V, Rosenspire $\mathrm{K}$, et al. Noninvasive evaluation of sympathetic nervous system in human heart by positron emission tomography. Circulation. 1990;82:457-464.

15. Raffel DM, Wieland DM. Assessment of cardiac sympathetic nerve integrity with positron emission tomography. Nucl Med Biol. 2001;28:541-559.

16. Tipre DN, Fox JJ, Holt DP, et al. In vivo PET imaging of cardiac presynaptic sympathoneuronal mechanisms in the rat. J Nucl Med. 2008;49:1189-1195.

17. Estorch M, Carrio I, Mena E, et al. Challenging the neuronal MIBG uptake by pharmacological intervention: effect of a single dose of oral amitriptyline on regional cardiac MIBG uptake. Eur J Nucl Med Mol Imaging. 2004;31:1575-1580.

18. Caldwell JH, Link JM, Levy WC, Poole JE, Stratton JR. Evidence for pre- to postsynaptic mismatch of the cardiac sympathetic nervous system in ischemic congestive heart failure. J Nucl Med. 2008;49:234-241.

19. Kenk M, Thackeray JT, Thorn SL, et al. Alterations of pre- and postsynaptic noradrenergic signaling in a rat model of adriamycin-induced cardiotoxicity. $J$ Nucl Cardiol. 2010;17:254-263.

20. Mazzadi AN, Pineau J, Costes N, et al. Muscarinic receptor upregulation in patients with myocardial infarction: a new paradigm. Circ Cardiovasc Imaging. 2009; 2:365-372. 